\title{
The effect of standard therapy on mean platelet volume in patients with chronic hepatitis $\mathrm{C}$
}

\author{
Ali Ugur Uslu', Bahattin Aydin², Sevket Balta ${ }^{3}$, Ozlem Yonem, \\ ${ }^{1}$ Department of Internal Medicine, Eskisehir Military Hospital, Eskisehir, Turkey \\ 2Department of Internal Medicine, Etimesgut Military Hospital, Ankara, Turkey \\ ${ }^{3}$ Department of Cardiology, Gulhane School of Medicine, Ankara, Turkey \\ ${ }^{4}$ Department of Gastroenterology, Faculty of Medicine, Cumhuriyet University, Sivas, Turkey \\ ${ }^{5}$ Department of Internal Medicine, Faculty of Medicine, Cumhuriyet University, Sivas, Turkey
}

Gastroenterology Rev 2016; 11 (3): 200-205

DOI: $10.5114 / p g .2016 .57942$

Key words: mean platelet volume, standard therapy, chronic hepatitis C, inflammation, liver.

Address for correspondence: Ali Ugur Uslu, Department of Internal Medicine, Eskisehir Military Hospital, Visnelik Mahalle Ataturk Cadde, 26020 Eskisehir, Turkey, phone: 5070431449, e-mail: drauuslu@gmail.com

\begin{abstract}
Introduction: Chronic hepatitis C (CHC) infection is a systemic disorder that can lead to liver inflammation, fibrosis, cirrhosis, and hepatocellular cancer. The mean platelet volume (MPV) is widely used as an inflammatory marker to evaluate the platelet function and the status of systemic inflammation.

Aim: To determine the pre- and post-treatment MPV values in CHC patients who were administered a 48-week antiviral therapy based on systemic inflammation.

Material and methods: We enrolled 28 patients, diagnosed with $\mathrm{CHC}$ genotype $1 \mathrm{~b}$, who received a 48-week antiviral therapy and attended regular follow-up, and 28 healthy individuals. In diagnosing CHC, a positive anti-HCV for a minimum duration of 6 months and a positive serum HCV RNA were accepted as the criteria. The patients were assigned to one of two groups based on their group 1 (pre-treatment values) and group 2 (post-treatment values) after 3 months therapy. We analysed and compared the blood samples of all of the groups.

Results: The MPV value was $8.89 \pm 1.20$ in group 1 and $8.00 \pm 1.07$ in group 2 , and $8.21 \pm 1.18$ in the control group. The value in group 1 was detected to be statistically significantly different from that in group 2 and the control group $(p<0.0001$, $p=0.045$, respectively). No statistically significant difference was observed between group 2 and the control group $(p=0.455)$.

Conclusions: The results of this study suggest that MPV could represent an inexpensive marker for use in assessing lowgrade inflammation in patients with $\mathrm{CHC}$.
\end{abstract}

\section{Introduction}

Hepatitis C virus (HCV) is one of the most important causes of chronic liver diseases. Hepatitis $C$ virus is known to have two different processes: acute or chronic, and is seen in approximately $3 \%$ of the population worldwide [1, 2]. Acute hepatitis cases may frequently lead to chronic hepatitis $\mathrm{C}(\mathrm{CHC})$ infection, liver fibrosis, cirrhosis, and hepatocellular cancer (HCC) $[3,4]$. Chronic hepatitis $C$ infection leads to systemic inflammation via steatosis, increased oxidative stress, and pro-inflammatory cytokines $[1,5]$.

A complete blood count is a relatively routine, inexpensive, practical and easy examination that gives additional information. Platelet activation is a link in the pathophysiology of diseases prone to thrombosis and inflammation [6]. The mean platelet volume (MPV) is the most commonly used among markers assessing the changes in platelet function and activation [7, 8]. The MPV also indicates inflammatory status, which is central to processes that are involved in inflammatory disease pathophysiology and endothelial dysfunction. Recently, MPV has been shown as an inflammatory marker in various inflammatory diseases [9-12]. A previous study reported that MPV levels are higher in $\mathrm{CHC}$ patients and that they are also correlated the activity index in these patients [13].

Based on the literature data, although the relation between the CHC patients and MPV levels were investigated in a previous study [13], the relation between 
MPV values and the standard therapy of CHC patients was not determined. Our study is the first to assess the MPV values before and after standard treatment in CHC patients.

\section{Aim}

The aim of the study was to determine the pre- and post-treatment MPV values in CHC patients and investigate its correlation with inflammation.

\section{Material and methods}

In our study, we enrolled 28 patients, diagnosed with $\mathrm{CHC}$ genotype $1 \mathrm{~b}$, who received 48-week pegylated interferon (Peg-IFN) $\alpha 2 \mathrm{a}$ and ribavirin (RBV) (800-1000 mg) and attended regular follow-up, and 28 healthy individuals. In diagnosing $\mathrm{CHC}$, a positive anti-HCV for a minimum duration of 6 months and a positive serum HCV RNA were accepted as the criteria. The patients were assigned to one of two groups based on their pre-treatment values and post-treatment values after 3 months therapy. According to the pre-treatment liver histopathological assessment, patients with a liver fibrosis score (LFS) between 0 and 2 were considered to have a low-grade fibrosis while those with an LFS between 3 and 6 had high-grade fibrosis [14].

Patients with diabetes mellitus, asthma, chronic obstructive pulmonary disease, cirrhosis, portal hypertension, renal dysfunction, peripheral and cerebral vascular disease, splenectomy, splenomegaly, malignity, and a history of alcohol use and/or smoking were excluded from the present study. The study was conducted in accordance with the Declaration of Helsinki, and local Ethics Committee approval was granted.

\section{Biochemical measurements}

Blood samples were drawn without stasis at 7-8 a.m. after 20 min of supine rest, following fasting for $\geq 12 \mathrm{~h}$. The blood was collected in tripotassium EDTA (7.2 mg) tubes. Haematological parameters, including haemoglobin $(\mathrm{Hb})$, white blood cells count (WBC), platelet count, and MPV, were analysed using an LH 780 analyser (Beckman Coulter Inc, Miami, Florida). We analysed the blood samples of all of the groups using an automatic blood counter within $1 \mathrm{~h}$ after venipuncture because a delay in the measurement could change the MPV values [15].

\section{Statistical analysis}

All statistical analyses were performed using SPSS version 14.0 (SPSS, Chicago, IL). Descriptive statistics were calculated for each of the variables (means and standard deviation (SD)). Paired sample $t$ test was used to evaluate the statistical data. Also, the non-parametric Wilcoxon Signed Ranks test was used to test for the differences between the related (paired) samples, and the Mann-Whitney $U$ test was used to investigate the differences between the independent samples. Spearman's correlation analysis was used to evaluate the correlation between data. A $p$ value $<0.05$ was considered statistically significantly two sided.

\section{Results}

There were 17 (60.7\%) male and 11 (39.3\%) female patients in the study, with a mean age of $53.6 \pm 12.9$ (range: $28-79$ years) years. In the control group, there were 13 (46.4\%) males and 15 (53.6\%) females, with a mean age of $46.4 \pm 17.4$ years (range: $20-77$ years). There were no statistically significant differences between the patients and the control subjects with respect to age and gender $(p=0.103$ and $p=0.288$, respectively). The rates of response to treatment, the post-histopathological assessment histologic activity index ( $\mathrm{HAl})$, and hepatic steatosis as detected by LFS and the abdominal ultrasonography (grade 1 ) are reported in Table I. The laboratory values in group 1 (pre-treatment) and group 2 (post-treatment) are given in Table II.

The comparison of certain parameters between group 1, group 2, and the control group is summarised in Tables III and IV. The MPV value was $8.89 \pm 1.20$ in group 1, $8.00 \pm 1.07$ in group 2, and $8.21 \pm 1.18$ in the control group. Baseline MPV values were higher in patients with $\mathrm{CHC}$ compared with those of controls (Figure 1) ( $p=0.045)$. Compared to group 2, group 1 had significantly different MPV levels (Figure 2) ( $p<0.001$ ). No statistically significant difference was observed between group 2 and the control group $(p=0.455)$. The

Table I. The baseline clinical characteristics of the patients

\begin{tabular}{lc} 
Treatment response & Result \\
\hline Sustained virological response, $n(\%)$ & $12(42.9)$ \\
\hline Non-sustained virological response, $n(\%)$ & $16(57.1)$ \\
\hline Abdominal ultrasonography: & $19(67.9)$ \\
\hline Normal, $n$ (\%) & $9(32.1)$ \\
\hline Hepatosteatosis, $n$ (\%) & $11(39.2)$ \\
\hline Liver fibrosis score 0-2, $n(\%)$ & $17(60.8)$ \\
\hline Liver fibrosis score 3-6, $n$ (\%) & \\
\hline Liver histopathological assessment: & $1.48 \pm 1.09$
\end{tabular}


Table II. Comparison of pre- and post-treatment in all patients with laboratory values

\begin{tabular}{lccc} 
Parameter & $\begin{array}{c}\text { Group 1 } \\
\text { Pre-treatment } \\
(n=28) \\
(\text { mean } \pm \text { SD) }\end{array}$ & $\begin{array}{c}\text { Group 2 } \\
\text { Post-treatment } \\
(n=28) \\
(\text { mean } \pm \text { SD) }\end{array}$ & P-value \\
\hline Alanine transaminase [IU/I] & $47.47 \pm 31.63$ & $26.40 \pm 20.63$ & 0.001 \\
\hline Aspartate transaminase [IU/I] & $45.88 \pm 28.60$ & $27.85 \pm 15.47$ & $<0.0001$ \\
\hline Alkaline phosphatase [IU/I] & $73.77 \pm 20.12$ & $75.92 \pm 20.11$ & 0.618 \\
\hline Gamma-glutamyl transferase [IU/I] & $40.59 \pm 27.78$ & $26.11 \pm 12.51$ & 0.005 \\
\hline Total bilirubin [mg/dl] & $0.93 \pm 0.32$ & $0.82 \pm 0.39$ & 0.216 \\
\hline Direct bilirubin [mg/dl] & $0.21 \pm 0.11$ & $0.18 \pm 0.12$ & 0.175 \\
\hline HCV-RNA [× 10 $/ \mathrm{IU} / \mathrm{ml}]$ & $49.61 \pm 5.03$ & $7.55 \pm 1.27$ & 0.011 \\
\hline Prothrombin time $[\mathrm{s}]$ & $11.50 \pm 1.39$ & - & - \\
\hline Albumin $[\mathrm{g} / \mathrm{dl}]$ & $3.87 \pm 0.40$ & - & 0.001
\end{tabular}

Table III. Pre-treatment and control group to compare the value of laboratory parameters

\begin{tabular}{lccc} 
Parameter & $\begin{array}{c}\text { Group 1 } \\
\text { (pre-treatment) } \\
(n=28) \\
(\text { mean } \pm \text { SD })\end{array}$ & $\begin{array}{c}\text { Controls } \\
(n=28) \\
(\text { mean } \pm \text { SD })\end{array}$ & $P$-value \\
\hline Haemoglobin $[\mathrm{g} / \mathrm{dl}]$ & $14.66 \pm 2.00$ & $13.66 \pm 1.66$ & 0.054 \\
\hline Leucocyte $\left.\left[\times 10^{9} /\right]\right]$ & $7.44 \pm 2.63$ & $7.05 \pm 1.62$ & 0.700 \\
\hline Platelet $\left[\times 10^{9} / \mathrm{l}\right]$ & $198.28 \pm 60.92$ & $258.00 \pm 44.88$ & $<0.0001$ \\
\hline Mean platelet volume $[\mathrm{fl}]$ & $8.89 \pm 1.20$ & $8.21 \pm 1.18$ & 0.045
\end{tabular}

Table IV. Post-treatment and control group to compare the value of laboratory parameters

\begin{tabular}{lccc} 
Parameter & $\begin{array}{c}\text { Group 2 } \\
\text { (post-treatment) } \\
(n=28) \\
(\text { mean } \pm \text { SD) }\end{array}$ & $\begin{array}{c}\text { Control } \\
(n=28) \\
(\text { mean } \pm \text { SD })\end{array}$ & $P$-value \\
\hline Haemoglobin $[\mathrm{g} / \mathrm{dll}]$ & $13.98 \pm 1.83$ & $13.66 \pm 1.66$ & 0.438 \\
\hline Leucocyte $\left[\times 10^{9} / \mathrm{l}\right]$ & $5.61 \pm 1.75$ & $7.05 \pm 1.62$ & 0.003 \\
\hline Platelet $\left[\times 10^{9} / \mathrm{l}\right]$ & $195.35 \pm 65.64$ & $258.00 \pm 44.88$ & $<0.0001$ \\
\hline Mean platelet volume $[\mathrm{fl}]$ & $8.00 \pm 1.07$ & $8.21 \pm 1.18$ & 0.455
\end{tabular}

All data mean $\pm S D$.

assessment of the abdominal ultrasound images revealed no statistically significant difference in the MPV values between the patients with and without grade 1 hepatic steatosis $(8.87 \pm 1.65$ and $8.90 \pm 0.96$, respectively; $p=0.693)$. The pre-treatment MPV values were not statistically significant between the patients who did and did not achieve complete treatment response (8.83 \pm 1.25 and $8.93 \pm 1.20$, respectively; $p=0.561$ ). There were statistically significant differences in the pre- and post-treatment MPV values between the patients who did and did not achieve complete treatment response ( $p=0.034$ and $p<0.001$, respectively) (Table V).

The pre-treatment histopathology assessment demonstrated no correlation between HAI, LFS, and MPV. The MPV did not show a statistically significant difference between the patients with low-grade and high-grade fibrosis $(8.75 \pm 1.24$ and $9.10 \pm 1.16$, respectively; $p=0.480)$. 


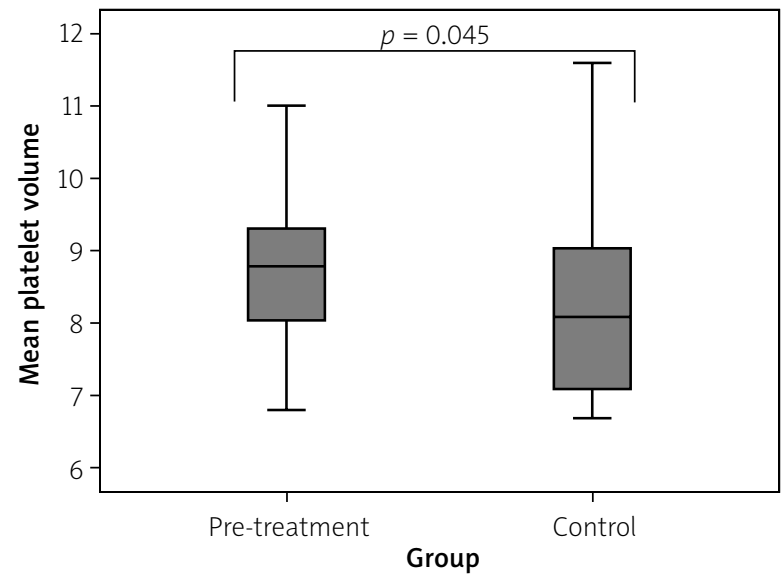

Figure 1 . There are statistically significant differences between baseline MPV values and the control group

\section{Discussion}

In our study, we have shown that pre-treatment MPV values were higher in CHC patients compare to post-treatment values, and that they were also higher compared to values in the control group. There were no differences between the post-treatment MPV value and the values in the control group. The complex effects of hepatitis $C$ on the liver have not been elucidated completely. Considering the controlling impact of the liver on the immune system, it directly or indirectly leads to local and systemic inflammation. As a result of the systemic inflammation, the effect on the precursor platelet cells in the bone marrow results in changes in MPV $[5,16]$.

The MPV shows variability between the presence of low-grade and high-grade inflammation. Recently, increased levels of MPV were demonstrated in many diseases [17], most of these are related to endothelial dysfunction on the basis of inflammation [18]. The higher MPV values are also considered to be a useful indicator of higher thrombocyte activity. The level of platelets is increased in cases of high-grade inflammation, leading to a reduction in the MPV level as a result of the migration of the majority of the large reactive platelets to inflammatory sites and intensive consumption of these platelets $[6,7]$. In the case of low-grade inflammation, an increase in the MPV level occurs as a result of the increased reactive immature platelets. Some studies reported that MPV were higher in patients with non-alcoholic fatty liver disease (NAFLD) [19], chronic hepatitis B (CHB) [12], and CHC [13]. Many noninvasive tests have been studied for diagnosis and determination of the LFS.

Non-alcoholic fatty liver disease is a chronic inflammatory disease with a pathogenesis involving oxidative stress and pro-inflammatory cytokines, which may lead

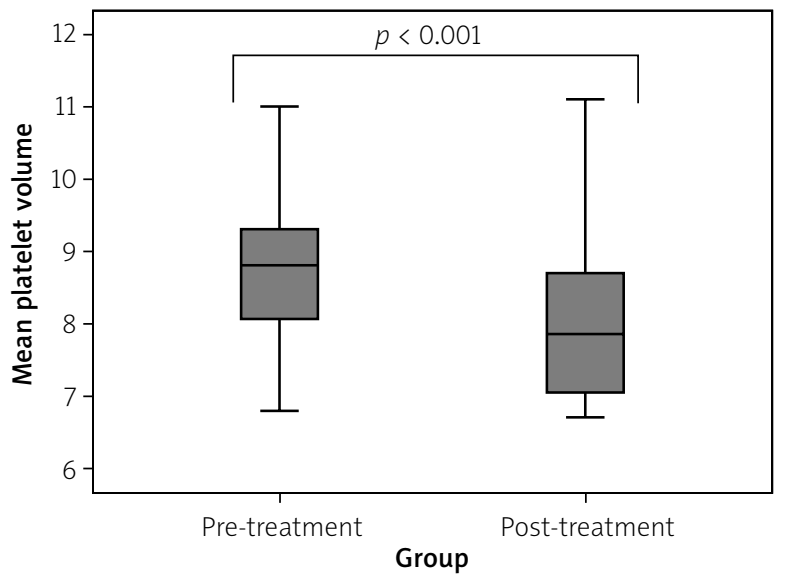

Figure 2. There are statistically significant differences between pre-treatment MPV values and the post-treatment group

Table V. Pre- and post-treatment MPV levels in relation to treatment response

\begin{tabular}{lccc} 
MPV & $\begin{array}{c}\text { Pre-treatment } \\
(\text { mean } \pm \text { SD) }\end{array}$ & $\begin{array}{c}\text { Post-treatment } \\
(\text { mean } \pm \text { SD })\end{array}$ & P-value \\
\hline $\begin{array}{l}\text { MPV in SVR } \\
(n=12)\end{array}$ & $8.83 \pm 1.25$ & $8.14 \pm 1.16$ & 0.034 \\
\hline $\begin{array}{l}\text { MPV in non-SVR } \\
(n=16)\end{array}$ & $8.93 \pm 1.20$ & $7.89 \pm 1.03$ & 0.001 \\
\hline & & & \\
$M P V-$ mean platelet volume, SVR - sustained virological response.
\end{tabular}

to non-alcoholic hepatic steatosis (NASH), fibrosis, and cirrhosis. Alkhouri et al. [10] demonstrated high MPV levels in NASH patients compared to steatosis patients, and patients with steatosis had higher MPV levels compared to those with a normal biopsy result. Celikbilek et al. [19] reported higher MPV levels in NAFLD patients relative to control subjects.

Chronic hepatitis B infection, like the CHC infection, is among the significant viral infections that can lead to chronic liver disease. It may result in liver inflammation, cirrhosis, and HCC. An increase in the MPV level occurs together with an increase in the production of immature platelets in the bone marrow in the case of $\mathrm{CHB}$ infection [12, 14, 20]. Turhan et al. [20] reported higher MPV in inactive CHB patients relative to healthy individuals. Ekiz et al. [12] demonstrated that high MPV levels were statistically significantly higher in CHB patients compared to the control group. They also showed that MPV was observed to be higher in patients with severe fibrosis relative to those without marked fibrosis. Ceylan et al. [14] showed no statistically significant difference in MPV level between the CHB patients with a liver fibrosis score of 0 to 2 and a LFS of 3 to 6 . The MPV may provide useful information to predict the degree of liver inflammation along with other markers [14]. 
Considering the effects of pro-inflammatory cytokines on platelet production and activation, studies in $\mathrm{CHC}$ patients mostly investigate the cytokine level. Interleukin-1 (IL-1), interleukin-6 (IL-6), and tumour necrosis factor $\alpha$ (TNF- $\alpha$ ) are among the progenitor cytokines that affect platelet production $[6,7,16]$. In the case of chronic liver disease, IL-6 production associated with secondary inflammation during the process of fibrosis and immature platelets are considered to increase the MPV level, suggesting a correlation with the grade of fibrosis $[6,12,16]$. Previous studies have shown that MPV levels were increased in $\mathrm{CHC}$ with inflammation and advanced fibrosis. Calculation of MPV along with the use of other markers may give further information about liver fibrosis severity in CHC [13]. Purnak et al. [13] reported statistically significantly higher MPV levels in patients relative to the control subjects. MPV was higher in patients with advanced fibrosis compared to those with mild fibrosis.

Proteins associated with hepatitis C infection stimulate the release of the pro-inflammatory cytokines. They are also increased in various types of chronic liver disease, including CHC. These cytokines are significantly involved in inflammation, regeneration, and fibrosis in the liver, and have an impact on the disease progression [16, 21]. Ueyama et al. [22] reported statistically significantly higher IL-6 levels in $\mathrm{CHC}$ patients relative to the control subjects. Huang et al. [23] demonstrated that serum IL-1 $\beta, \mathrm{IL}-6$, and TNF- $\alpha$ levels are elevated in patients with HCV-related liver diseases, especially in liver cirrhosis. These levels reflect hepatic dysfunction better than liver inflammation parameters, which might explain the higher serum concentrations of cytokines in liver cirrhosis patients. In another study, Ivashkin et al. [24] reported a reduction in post-treatment TNF- $\alpha$ levels relative to the pre-treatment values in CHC patients. Peg-IFN and RBV therapy provides a reduction in local and/or systemic inflammation due to the regression of necro-inflammation and liver injury in $\mathrm{CHC}$ patients.

These above studies have shown that some inflammatory markers affecting platelet function may be changed after standard therapy in patients with $\mathrm{CHC}$. Consequently, we aimed to investigate the MPV levels as platelet activity markers after standard therapy in patients with $\mathrm{CHC}$. We reported that there were statistically significant differences of MPV values before and after standard therapy. This study has some limitations; they include the small number of patients, the retrospective nature of the study, the fact that the correlation between the cytokine levels and MPV was not investigated, and the absence of assessment of histopathology investigations on post-treatment liver biopsies.

\section{Conclusions}

We suggest that MPV is a simple and an inexpensive marker that may be used in assessing low-grade chronic inflammation in patients with $\mathrm{CHC}$. Further prospective randomised studies are needed to elucidate the significance of MPV for CHC patients.

\section{Conflict of interest}

The authors declare no conflict of interest.

\section{References}

1. Irshad M, Mankotia DS, Irshad K. An insight into the diagnosis and pathogenesis of hepatitis C virus infection. World J Gastroenterol 2013; 19: 7896-909.

2. Boroń-Kaczmarska A. Pathomechanism of metabolic disorders in HCV infection. Przegl Epidemiol 2006; 60: 701-5.

3. Tai WC, Hu TH, Wang JH, et al. Clinical implications of alpha-fetoprotein in chronic hepatitis C. J Formos Med Assoc 2009; 108: 210-8.

4. Kupś-Rzepecka J, Gołąbek V, Kiciński P, Woźniakowska-Gęsicka T. Efficacy and safety of pegylated interferon alpha-2b and ribavirin in chronic hepatitis $C$ in children. Prz Gastroenterol 2010; 5: 341-8

5. Adinolfi LE, Zampino R, Restivo L, et al. Chronic hepatitis C virus infection and atherosclerosis: clinical impact and mechanisms. World J Gastroenterol 2014; 20: 3410-7.

6. Gasparyan AY, Ayvazyan L, Mikhailidis DP, Kitas GD. Mean platelet volume: a link between thrombosis and inflammation? Curr Pharm Des 2011; 17: 47-58.

7. Korkmaz S, Uslu AU, Sahin S, et al. Is there a link between mean platelet volume and thrombotic events in antiphospholipid syndrome? Platelets 2013; 7104: 1-5.

8. Aktas G, Alcelik A, Tekce BK, et al. Red cell distribution width and mean platelet volume in patients with irritable bowel syndrome. Prz Gastroenterol 2014; 3: 160-3.

9. Yüksel O, Helvaci K, Başar O, et al. An overlooked indicator of disease activity in ulcerative colitis: mean platelet volume. Platelets 2009; 20: 277-81.

10. Alkhouri N, Kistangari G, Campbell C, et al. Mean platelet volume as a marker of increased cardiovascular risk in patients with nonalcoholic steatohepatitis. Hepatology 2012; 55: 331.

11. Balta S, Demirkol S, Cakar M, Celik T. Mean platelet volume as a surrogate marker of low-grade inflammation in osteoarthritis. Platelets 2014; 25: 643-4.

12. Ekiz F, Yüksel O, Koçak E, et al. Mean platelet volume as a fibrosis marker in patients with chronic hepatitis B. J Clin Lab Anal 2011; 25: 162-5.

13. Purnak T, Olmez S, Torun S, et al. Mean platelet volume is increased in chronic hepatitis $C$ patients with advanced fibrosis. Clin Res Hepatol Gastroenterol 2013; 37: 41-6.

14. Ceylan B, Mete B, Fincanci M, et al. A new model using platelet indices to predict liver fibrosis in patients with chronic hepatitis B infection. Wien Klin Wochenschr 2013; 125: 453-60.

15. Balta S, Demirkol S, Kucuk U, et al. Mean platelet volume may be related to the degree of coronary collateral circulation. Eur Rev Med Pharmacol Sci 2013; 17: 707-8. 
16. Zampino R, Marrone A, Restivo L, et al. Chronic HCV infection and inflammation: clinical impact on hepatic and extra-hepatic manifestations. World J Hepatol 2013; 5: 528-40.

17. Balta S, Demirkol S, Kucuk U, Unlu M. Hemostatic markers can be pivotal roles of risk factors for new-onset atrial fibrillation. Platelets 2014; 25: 554-5.

18. Demirkol S, Balta S, Unlu M, et al. Evaluation of the mean platelet volume in patients with cardiac syndrome $X$. Clinics (Sao Paulo) 2012; 67: 1019-22.

19. Celikbilek M, Gürsoy S, Deniz K, et al. Mean platelet volume in biopsy-proven non-alcoholic fatty liver disease. Platelets 2013; 24: 194-9.

20. Turhan O, Coban E, Inan D, Yalcin AN. Increased mean platelet volume in chronic hepatitis B patients with inactive disease. Med Sci Monit 2010; 16: CR202-5.

21. Larrubia JR, Moreno-Cubero E, Lokhande MU, et al. Adaptive immune response during hepatitis $C$ virus infection. World J Gastroenterol 2014; 20: 3418-30.

22. Ueyama M, Nakagawa M, Sakamoto N, et al. Serum interleukin-6 levels correlate with resistance to treatment of chronic hepatitis $C$ infection with pegylated-interferon-alpha2b plus ribavirin. Antivir Ther 2011; 16: 1081-91.

23. Huang YS, Hwang SJ, Chan CY, et al. Serum levels of cytokines in hepatitis C-related liver disease: a longitudinal study. Zhonghua Yi Xue Za Zhi (Taipei) 1999; 62: 327-33.

24. Ivashkin VT, Mammaev SN, Lukina EA, et al. Cytokine system in patients with chronic hepatitis $C$ during treatment with interferon-alfa. Ter Arkh 2002; 74: 37-41.

Received: 22.04 .2015

Accepted: 17.06.2015 